\title{
High performance liquid chromatography of biliprotein from Bangia atropurpurea 'Conchocelis'
}

\author{
Hong-Nong Chou \& Jiunn-Ming Jeng \\ Institute of Fisheries Science, National Taiwan University, Taipei, Taiwan, Republic of China
}

Key words: anion exchange chromatography, reverse phase high performance liquid chromatography, phycoerythrin charged-isomers, biliprotein subunits, Bangia atropurpurea, conchocelis

\begin{abstract}
Gel-filtration-purified R-phycoerythrin and phycocyanin from the filamentous phase of Bangia atropurpurea were subjected to high performance anion exchange chromatographic separation. The purified R-phycoerythrin was a mixture of three charged-isomers that were well resolved in a Bio-Gel MA7P column by elution with a $\mathrm{NaCl}$ gradient in phosphate buffer. These were tentatively called phycoerythrin charged-isomers and were also separable from phycocyanin and allophycocyanin by the same system. Hence, it is suggested that the ion exchange chromatographic and the sample preparation methods presented in this report can be used to distinguish the various natures of biliproteins in red algae in addition to the polyacrylamide gel electrophoresis technique. Isomers of R-phycoerythrin have the same absorption and emission spectra and were studied for their subunit compositions by a PRP-3 reverse phase column chromatography. All three charged-isomers have the same $\alpha$ and $\beta$ subunits in common but differ in their $\gamma$ subunit.
\end{abstract}

\section{Introduction}

Photosynthetic accessory pigments of phycobiliproteins have been extensively studied in the blue-green alga and in some species of the red alga (O'hEocha, 1965). However, there are conflicts between the identifications of various types of pigments in identical species of algae (Kikuchi et al., 1979; Zeng et al., 1984), as well as for criteria of biliprotein purity (Gantt, 1981; Glazer \& Hixon, 1975; Glazer et al., 1982). Phycobiliproteins have been purified using various methods including ion exchange chromatographies, reverse phase partition chromatography, gel filtration, preparative electrophoresis and even gradient centrifugation (Swanson \& Galzer, 1990). Most of them were performed under denaturing condition. Thus, the isolated proteins from different purification methods showed spectrophotometric absorption discrepancies.

Gantt \& Lipschultz (1974) found 2 or 3 close bands for B-phycoerythrins in the electrophoretic separation of Porphyridium cruentum biliproteins and referred to them as charged-isomers of B-phycoerythrin because of their similarity in molecular weight and their difference in charge density. Swanson \& Glazer (1990) found 3 close peaks corresponding to the different $\gamma$ subunits in the gelfiltration-purified $P$. cruentum B-phycoerythrin by reverse phase high performance liquid chromatography. However, they did not point out any correlation between the different $\gamma$ subunits and B-phycoerythrin charged-isomers. In an earlier study, Yu et al. (1981) reported that two components of R-phycoerythrin were resolved in native form by polyacrylamide gel electrophoresis in 
both Callithamnion roseum and $C$. byssoids. They also mentioned that these two components were different in their patterns on SDS-polyacrylamide gel electrophoresis. One of the components contained polypeptides of 33 and $19 \mathrm{KD}$, whereas the other component contained polypeptides of 30 and $19 \mathrm{KD}$. It was speculated that the 30 and $33 \mathrm{KD}$ polypeptides were $\gamma$ and $\gamma^{\prime}$ subunits. On SDS-polyacrylamide gel electrophoresis, Talarico (1990) also revealed the existence of $\alpha, \beta, \gamma$ and $\gamma^{\prime}$ subunits in Audouinella saviana R-phycoerythrin. Klotz \& Glazer (1985) studied the types and numbers of phycobilins on the subunits of phycoerythrin and revealed that the trypsindigested $\gamma$ subunit of Gastroclonium coulteri contained 5 different phycobilin-carrying peptides. Because the $\gamma$ subunit was known to contain 4 phycobilins from previous spectroscopic studies, it was speculated that there were more than two types of phycoerythrin $\gamma$ subunit in G. coulteri. However, Swanson \& Glazer (1990) in subsequent HPLC studies found that there was only one peptide peak corresponding to the $\gamma$ subunit.

In this report, we used anion exchange chromatography and reverse phase high performance liquid chromatography for the analysis of phycoerythrin charged-isomers and their subunits from the filamentous phase ('Conchocelis') of Bangia atropurpurea. In preliminary experiments, it was found that the charged-isomers observable through anion exchange chromatography differed according to algal species. The ion exchange chromatogram can thus be used as a 'finger-print' identification in Rhodophyta classification.

\section{Materials and methods}

\section{Chemicals}

HPLC-grade acetonitrile and isopropanol were obtained from Alps Chem Co. (Taipei, Taiwan) and TFA from Sigma Chemical Co. (St. Louis, MO). Water for HPLC was made from Milli-Q Reagent Water System (Nihon Millipore Kogyo K. K., Yonezawa, Japan) of double-distilled water. All other chemicals were of reagent grade.
Algal specimens and culture condition

Stock culture of filamentous phase of Bangia atropurpurea (Roth) C. Agardh was kindly given by Dr Y. M. Chiang, Institute of Oceanography, National Taiwan University. The species was also identified by Dr Y. M. Chiang. The filamentous plants ('Conchocelis') were originally germinated from a single carpospore of $B$. atropurpurea by the method similar to that used for Porphyra species (Chiang \& Wang, 1980). Filaments were propagated by fragmentation and grew into colonies of filamentous clusters in SWM-III medium (Chen et al., 1969). Several 'Conchocelis' colonies of the stock culture were blended into fragments of 200 to $300 \mu \mathrm{m}$ long by a sterile grinder for $30 \mathrm{~s}$ at low speed and then inoculated into fresh medium. The 'Conchocelis' suspension was incubated at $18{ }^{\circ} \mathrm{C}$ under fluorescent light $\left(26.3 \mu \mathrm{mol} \mathrm{m}^{-2} \mathrm{~s}^{-1}\right.$ for 14h:10 L:D. Mass cultures of the filamentous plants were maintained in 201 polycarbonate carboys (Nalgene Labware, Rochester, NY) with continuous aeration by aquarium air pumps. After a month of incubation, plants were harvested with a plankton net. Removal of excess water was done by filtration on a Buchner funnel using suction.

\section{Preparation of pure $R$-phycoerythrin}

Wet plants were frozen in liquid nitrogen and then ground in a mortar. Plant fragments were extracted by continuous stirring in $10 \mathrm{mM} \mathrm{K}$ phosphate (pH7.0, $1 \mathrm{mM} \mathrm{NaN}_{3}$ ) in an ice-bath. After centrifugation at $7000 \mathrm{~g}$ for $20 \mathrm{~min}$ at $4{ }^{\circ} \mathrm{C}$, the supernatant was brought to $30 \%$ saturation of ammonium sulfate. The pigmented solution was separated from the precipitate by centrifugation and was brought to $65 \%$ saturation of ammonium sulfate to precipitate the biliproteins. The precipitate, collected by centrifugation, was resuspended in $10 \mathrm{mM}$ phosphate buffer, and dialyzed overnight at $4{ }^{\circ} \mathrm{C}$ against 100 sample volumes of the same buffer. The protein solution was applied to a column $(2.5 \times 120 \mathrm{~cm})$ of Sephadex G-200 (Pharmacia Fine Chemicals, Uppsala, Sweden) preequilibrated with the same buffer. The column was washed with $10 \mathrm{mM} \mathrm{K}$-phosphate with a flow rate of $15 \mathrm{ml} \mathrm{h}^{-1}$ and the ef- 
fluents as collected in $3.75 \mathrm{ml}$ fractions. Elution was monitored by absorption at $280,496,615$, and $650 \mathrm{~nm}$ using a Hitachi UV-Visible spectrometer, Model 150-20 (Hitachi Ltd., Tokyo, Japan). Repeated gel-filtrations were required for the purification of R-phycoerythrin and other phycobiliproteins.

Anion exchange chromatography of $R$-phycoerythrin and other phycobiliproteins

High performance ion exchange chromatography and reverse phase chromatography were carried out using two L-6000 pumps, one L-5000 LC controller for gradient program setting, one L-4200 UV-VIS Detector, and one D-2000 Chromato-integrator (Hitachi Ltd., Tokyo, Japan). The column used in the ion exchange chromatography was Bio-Gel MA7P ( $7.8 \times 50 \mathrm{~mm}$, Bio-Rad Laboratories, Richmond, CA). The mobile phase was composed of two buffers, A (10 mM K-phosphate, pH7.0) and B (10 mM K-phosphate, $1 \mathrm{M} \mathrm{NaCl}, \mathrm{pH}$ 7.0). Flow rate was $1 \mathrm{ml} \mathrm{min}^{-1}$. R-phycoerythrin chargedisomers were separated using a gradient from 0 to $70 \%$ of B in 14 minutes. Phycocyanin and allophycocyanin from the gel-filtration-purified fractions were separated using a gradient from 0 to $100 \%$ of B in 10 minutes. Elutions of proteins, phycoerythrins and phycocyanins were monitored at 280,496 , and $615 \mathrm{~nm}$, respectively.

\section{Reverse phase chromatography}

Reverse phase HPLC was performed using a PRP-3 column, $4.1 \times 150 \mathrm{~mm}$, (Hamilton Co., Reno, NV). B. atropurpurea R-phycoerythrin charged-isomers, isolated by anion exchange chromatography and then dialyzed against $10 \mathrm{mM}$ potassium phosphate buffer, were injected onto the column previously equilibrated in $70 \%$ trifluoroacetic acid (TFA, $1 \%$ ) water (solution A), and $30 \% 2: 1$ acetonitrile:isopropanol containing $1 \%$ TFA (solution B). Proteins were eluted from the column at a flow rate of $1.5 \mathrm{ml} \mathrm{min}{ }^{-1}$ and in a linear gradient from $30 \%$ to $100 \%$ of B in 10 minutes. Elutions of the resolved peptide subunits were detected by absorption at $280 \mathrm{~nm}$.

\section{Results}

Purification of R-phycoerythrin by gel filtration chromatography

About $20 \mathrm{~g}$ of dry algal material was obtained from 201 culture of $B$. atropurpurea 'Conchocelis'. The ratio of absorption at $565 \mathrm{~nm}$ to that at $280 \mathrm{~nm}\left(\mathrm{~A}_{565} / \mathrm{A}_{280}\right)$ was 1.54 for the algal phosphate extract and was used as an index for the relative contents of phycoerythrin in the solution. The Sephadex G-200 gel filtration chromatogram of the pigments is shown in Fig. 1. Absorption at $280 \mathrm{~nm}, 565 \mathrm{~nm}, 615 \mathrm{~nm}$, and $650 \mathrm{~nm}$ follows the elution of protein, phycoerythrin, phycocyanin, and allophycocyanin, respectively. The ratio $A_{565} / A_{280}$ for fractions 95 to 105 corresponding to phycoerythrin was larger than 3.5 after the first gel-filtration. These fractions were concentrated by precipitation in $65 \%$ saturation of ammonium sulfate and dialyzed against $10 \mathrm{mM}$ phosphate buffer. After the second gel-filtration, the $\mathrm{A}_{565} /$ $A_{280}$ ratio of phycoerythrin was greater or equal to 5.2. From this value and the UV-visible spectrum, it was determined as a highly pure sample of R-phycoerythrin (Gantt, 1969; Ogawa et al., 1991; Talarico, 1990). A purple-blue pigment (absorption at $615 \mathrm{~nm}$ ) was eluted after R-phycoerythrin (Fig. 1). Spectroscopic analysis demonstrated that fraction \# 117 contained phycocyanin and allophycocyanin. This was further confirmed by ion exchange chromatographic and spectroscopic studies. Another red pigment (detected by $565 \mathrm{~nm}$ absorption) was eluted in fractions 140 to 155 . This pigment was identified as r-phycoerythrin by comparison of its spectrum with the one reported by Gantt \& Lipschultz (1980).

\section{Anion exchange chromatography}

Gel-filtration purified pigments were analyzed by HPLC using a Bio-Gel MA7P column, a nonporous polyethylenimine weak anion exchanger. The highly purified R-phycoerythrin (Fr. \#100 in Fig. 1) was composed of three different protein molecules eluted from the column at different retention times $(6.36,7.50$, and $8.09 \mathrm{~min})$ by a gradient of increasing $\mathrm{NaCl}$ concentration. Because 


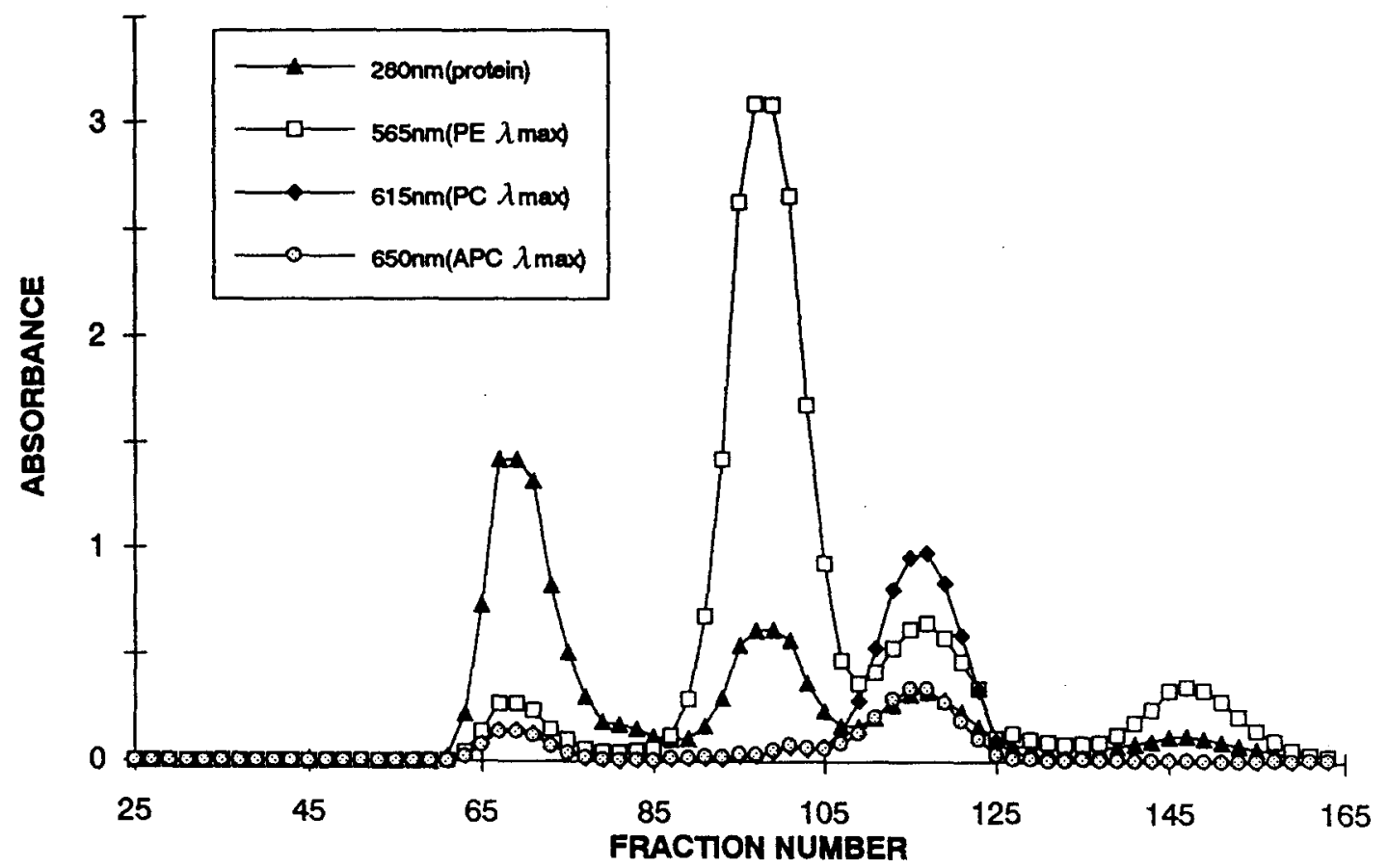

Fig. 1. Separation of Bangia atropurpurea phycobiliproteins by Sephadex G-200 column chromatography. Each fraction was monitored by a spectrometer for the absorption of specified light wavelengths.

elution time from this column is based on the charge density of molecules, the different components of R-phycoerythrin were tentatively referred to as the charged-isomers of the pigment and were assigned PE-I, PE-II, and PE-III, respectively, according to their elution sequence. All three R-phycoerythrin charged-isomers have the same spectra as the one of the gel-filtration purified phycoerythrin. Native polyacrylamide gel electrophoresis of these three isomers also showed close bands that were not separated in the the same PAGE analysis of the Sephadex purified sample.

Fraction \# 117, representing the blue pigment purified by repeated Sephadex G-200 column separation was also analyzed by ion exchange chromatography. The result showed that it contains one major (Rt. $3.96 \mathrm{~min}$ ) and one minor components (Rt. $6.00 \mathrm{~min}$ ). From the absorption spectra of these components, it was found that the major blue pigment was R-phycocyanin and the minor one was allophycocyanin (Gantt \& Lipschultz, 1974). Fraction \# 149 of r-phycoerythrin gave one broad peak at both 280 and
$496 \mathrm{~nm}$ on the Bio-Gel MA7P anion exchange chromatograms. This result showed a rather complex composition of r-phycoerythrin.

\section{Reverse phase chromatography}

Although various types of reverse phase columns including silica based $\mathrm{C} 4$ or $\mathrm{C} 18$ columns were tested for biliprotein subunit analysis, it was found that the Hamilton PRP-3 column gave a better result in our laboratory. The separation was comparable to that obtained with a C18 (Padgett \& Krogmann, 1987) and C4 column (Swanson \& Glazer, 1990). Phycoerythrin charged-isomers that were purified from preparative anion exchange chromatography were further studied for their subunit compositions by PRP-3 reverse phase chromatography. All three charged-isomers of R-phycoerythrin contain three different subunits, $\alpha, \beta$, and $\gamma$ (Fig. 2) as reported by Swanson \& Glazer (1990) and Yu et al. (1981). Identification of the subunits was done by comparing their absorption spectra with those reported in the literature and from their molecular weights deter- 


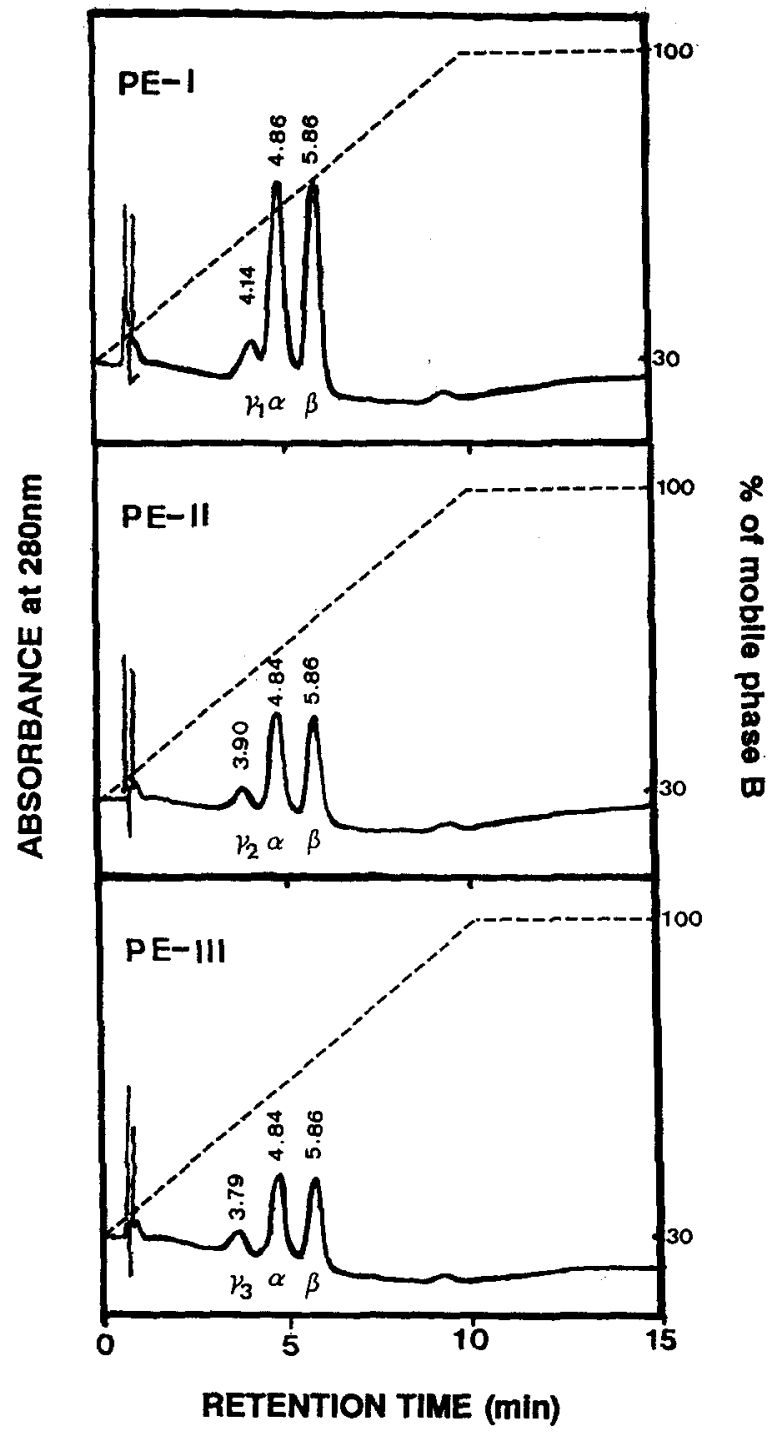

Fig. 2. Reverse phase chromatograms of R-phycoerythrin charged-isomers isolated from Bangia atropurpurea, revealing the $\alpha, \beta$, and $\gamma\left(\gamma_{1}, \gamma_{2}\right.$, and $\left.\gamma 3\right)$ subunits in the proteins. Column: Hamilton PRP-3 $(4.1 \times 150 \mathrm{~mm})$; mobile phase: solution $\mathrm{A}$, deionized water $/ 0.1 \%$ TFA; solution $\mathrm{B}$, acetonitrileisopropanol $(2: 1, \mathrm{v} / \mathrm{v}) / 0.1 \%$ TFA, flow rate $1.5 \mathrm{ml} \mathrm{min}{ }^{-1}$; dashed lines show the percentage of solution $\mathrm{B}$ in gradient elutions.

mined by SDS-PAGE. The same retention times for the $\alpha$ subunit $(4.86 \mathrm{~min})$ and the $\beta$ subunit $(5.86 \mathrm{~min}$ ) of the different isomers were obtained. Hence it was judged that the $\alpha$ or the $\beta$ subunits in different R-phycoerythrin charged-isomers were all identical. However, in the same analysis, the $\gamma$ subunits from different isomers showed different retention times. It was concluded that the different $\gamma$ subunits in phycoerythrin were responsible for the different charged-isomers found. Still, the different $\gamma$ subunits were not resolvable by HPLC or SDS-PAGE when the injected sample was a mixture of charged-isomers.

\section{Discussion}

There are reports on the charged- or size isomers of B-phycoerythrin from $P$. cruentum (Gantt \& Lipschultz, 1974; Glazer \& Hixon, 1977) and Rhodella violacea (Koller \& Wehrmeyer, 1975; Morschel et al., 1980). Callithamnion roseum and C. byssoides were also found to contain two $\mathrm{R}$-phycoerythrin isomers that were different in their $\gamma$ subunit (Yu et al., 1981). Our observations of R-phycoerythrin charged-isomers in B. atropurpurea are not unprecedented. However, phycoerythrin isomers have never been resolved by ion exchange chromatography. In subsequent studies using anion exchange chromatography for native biliprotein analysis, it was found that the existence of phycoerythrin charged-isomers was very common in red algae (Chou \& Jeng, unpublished data). It was wondered why there were only a few reports describing the multiple natures of phycoerythrin in red algae. One explaination may be the low resolution of the methods generally applied for phycobiliprotein research. The methodology described in this report will facilitate the screening of different phycobiliproteins in different algae. Similarity or dissimilarity of the isomer profile may give some phylogenic correlation data in systematic taxonomy.

\section{Acknowledgements}

We express our sincere thanks to Dr Y.M. Chiang for his gift of Bangia atropurpurea 'conchocelis' stock culture and advises in growing them. This study was supported by the National Science Council, Republic of China. 


\section{References}

Chen, L. C. M., T. Edelstein \& J. McLachlan, 1969. Bonnemaisonia hamifera in nature and in culture. J. Phycol. 5: 211-220.

Chiang, Y. M. \& J. C. Wang, 1980. A study on the production of conchosporangia in the conchocelis phase of Porphyra angusta Okamura et Ueda. Phycologia 19: 20-24.

Gantt, E., 1969. Properties and ultrastructure of phycoerythrin from Porphyridium cruentum. Plant Physiol. 44: 16291638.

Gantt, E., 1981. Phycobilisomes. Ann. Rev. Pl. Physiol. 32: 327-347.

Gantt, E. \& C. A. Lipschultz, 1974. Phycobilisomes of Porphyridium cruentum: pigment analysis. Biochemistry 13: 2960-2966.

Gantt, E. \& C. A. Lipschultz, 1980. Structure and phycobiliprotein composition of phycobilisomes from Griffithsia pacifica (Rhodophyceae). J. Phycol. 16: 394-398.

Glazer, A. N. \& C. S. Hixon, 1975. Characterization of Rphycocyanin. J. Biol. Chem. 250: 5487-5495.

Glazer, A. N., J. A. West \& C. Chan, 1982. Phycoerythrins as chemotaxonomic markers in red algae: a survey. Biochem. sys. Ecol. 10: 203-215.

Kikuchi, R., K. Ashida \& S. Hirao, 1979. Phycobilins in different color types of Porphyra yezoensis Ueda. Nippon Suisan Gakkaishi 45: 1461-1464.

Klotz, A. V. \& A. N. Glazer, 1985. Characterization of the bilin attachment sites in R-phycoerythrin. J. biol. Chem. 260: $4856-4863$.
Koller, K.-P. \& W. Wehrmeyer, 1975. B-phycoerythrin from Rhodella violace a characterization of two isoproteins. Arch. Microbiol. 104: 255-261.

Morschel, E., W. Wehrmeyer \& K.-P. Koller, 1980. Biliprotein assembly in the disc-shaped phycobilisomes of Rhodella violacea: Electron microscopical and biochemical analysis of B-phycoerythrin and B-phycoerythrin-C-phycocyanin aggregate. Eur. J. Cell Biol. 21: 319-327.

Ogawa, H., H. Mizuno, T. Saito, Y. Yamada, T. Oohusa \& N. Iso, 1991. Effects of pH on the conformation of phycoerythrin from nori Porphyra sp.. Nippon Suisan Gakkaishi 57: 899-903.

O'hEocha, C., 1965. Biliproteins of algae. Ann. Rev. Pl. Physiol. 16: 415-434.

Padgett, M. P. \& D. W. Krogmann, 1987. Large scale preparation of pure phycobiliproteins. Photosyn. Res. 11: 225235 .

Swanson, R. V. \& A. N. Glazer, 1990. Separation of phycobiliprotein subunits by reverse-phase highpressure liquid chromatography. Analyt. Biochem. 188: 295-299.

Talarico, L., 1990, R-phycoerythrin from Audouinella saviana (Nemaliales, Rhodophyta): Ultrastructural and biochemical analysis of aggregates and subunits. Phycologia 29: 292-302.

Yu, M. H., A. N. Glazer, K. G. Spencer and J. A. West, 1981. Phycoerythrins of the red alga Callithamnion. Plant Physiol. 68: 482-488.

Zeng F. J., Z. X. Yang \& L. J. Jiang, 1984. Isolation and characterization of R-phycocyanin from Polysiphonia urceolata. Hydrobiologia 116/117: 594-596. 